\title{
Expression of CD44 variants and prognosis in oesophageal squamous cell carcinoma
}

T Gotoda, Y Matsumura, H Kondo, H Ono, A Kanamoto, H Kato, H Watanabe, Y Tachimori, Y Nakanishi, T Kakizoe

\begin{abstract}
Background-The CD44 variant (CD44v) isoforms have been noted as markers for tumour metastasis and prognosis in several adenocarcinomas.

Aims-To investigate whether CD44v, especially the CD44v2 (v2) isoform, may be a useful prognostic factor for patients with oesophageal squamous cell carcinoma, using a recently developed monoclonal antibody against a v2 epitope.

Patients-233 patients (211 men and 22 women; mean age 61.9 years), with oesophageal squamous cell carcinomas curatively removed without additional treatment between 1987 and 1996 at the National Cancer Center Hospital, were analysed for CD44v expression.
\end{abstract}

Methods-The expression of CD44v was evaluated immunohistochemically using monoclonal antibodies against epitopes of the standard and variant protein, in paraffin embedded oesophageal squamous cell carcinoma tissue from 233 patients who had undergone cervical, mediastinal, and abdominal lymphadenectomy (three field dissection) for oesophagectomy. The data were evaluated for any correlation with clinicopathological indices or prognosis.

Results-Although total CD44 and CD44v6 (v6) were respectively observed in $99 \%$ and $97 \%$ of the cancer specimens, the expression of v2 was only $30 \%$. Patients whose tumours were $\mathbf{2} 2$ positive had a significantly better prognosis than those whose tumours were $\mathbf{v} 2$ negative ( $p=$ 0.031). Furthermore, in patients without lymph node metastasis, $\mathbf{v} 2$ positivity alone was a significant independent factor of prognosis (relative risk of death associated with v2 negativity, $4.7 ; p=0.037$ ) in multivariate analysis.

Conclusions-These results indicate that v2 is a useful marker for clinical prognosis in patients with oesophageal squamous cell carcinoma. Particularly in patients without lymph node metastasis, v2 status may thus have implications for the use of adjuvant chemotherapy and/or radiotherapy in patients with oesophageal cancer at an early stage.

(Gut 2000;46:14-19)

Keywords: CD44 variants; oesophageal squamous cell carcinoma; metastasis; prognosis;

immunohistochemistry
CD44 is a heavily glycosylated cell surface molecule which is involved in cell-cell and cellmatrix interactions ${ }^{12}$ and mediates several functions, such as extracellular matrix cell adhesion, ${ }^{34}$ lymphocyte homing, ${ }^{56} \mathrm{~T}$ cell activation, ${ }^{7}$ and tumour metastasis. ${ }^{78}$ The CD44 family of polymorphic transmembrane glycoproteins is encoded by a complex gene $^{79-12}$ which occupies a stretch of $60-80 \mathrm{~kb}$ assigned to the human chromosomal locus $11 \mathrm{p} 13$. Its human form is composed of at least 21 exons, 10 of which are constitutively expressed on almost all cell types, to produce a heavily glycosylated $85-90 \mathrm{kDa}$ isoform known as standard form CD44. The remaining exons can be alternatively spliced in various combinations, and their products are incorporated into the polypeptide backbone encoded by the standard form exons. This results in a large array of protein isoforms (CD44v), which are differentially expressed in various tissues and at various stages in development. ${ }^{13} 14$

It was recently shown that the expression of v6, one variant isoform of CD44, distinguished metastatic from non-metastatic pancreatic carcinoma cell lines in the rat. ${ }^{8}$ Evidence that v6 itself has a role in metastasis came from the demonstration that transfection with cDNA encoding this isoform converted non-metastatic carcinoma rat cells into metastatic cells. ${ }^{8}$ Although the functions of CD $44 \mathrm{v}$ isoforms in humans remain unclear, it is thought that they play an important role in the growth and metastasis of several kinds of tumours. ${ }^{15-19}$ However, there have been no reports on the relation between $C D 44 \mathrm{v}$ expression and oesophageal cancer. We analysed the correlations between clinicopathological indices and immunohistochemical staining of surgical specimens curatively resected from oesophageal squamous cell carcinomas, using anti-v6 antibody. In addition, we evaluated whether monoclonal antibody against an epitope encoded by exon 7 (v2) ${ }^{20}$ could be a prognostic factor, because several molecular biological studies have indicated that abnormal expression of v2 occurs in various types of human malignancies ${ }^{21} 22$ and no study has yet evaluated it in human oesophageal cancer.

Abbreviations used in this paper: $\mathrm{CD} 44 \mathrm{~s}, \mathrm{CD} 44$ standard; CD44v, CD44 variant; CD44v2, CD44 variant 2; CD44v6, CD44 variant 6. 

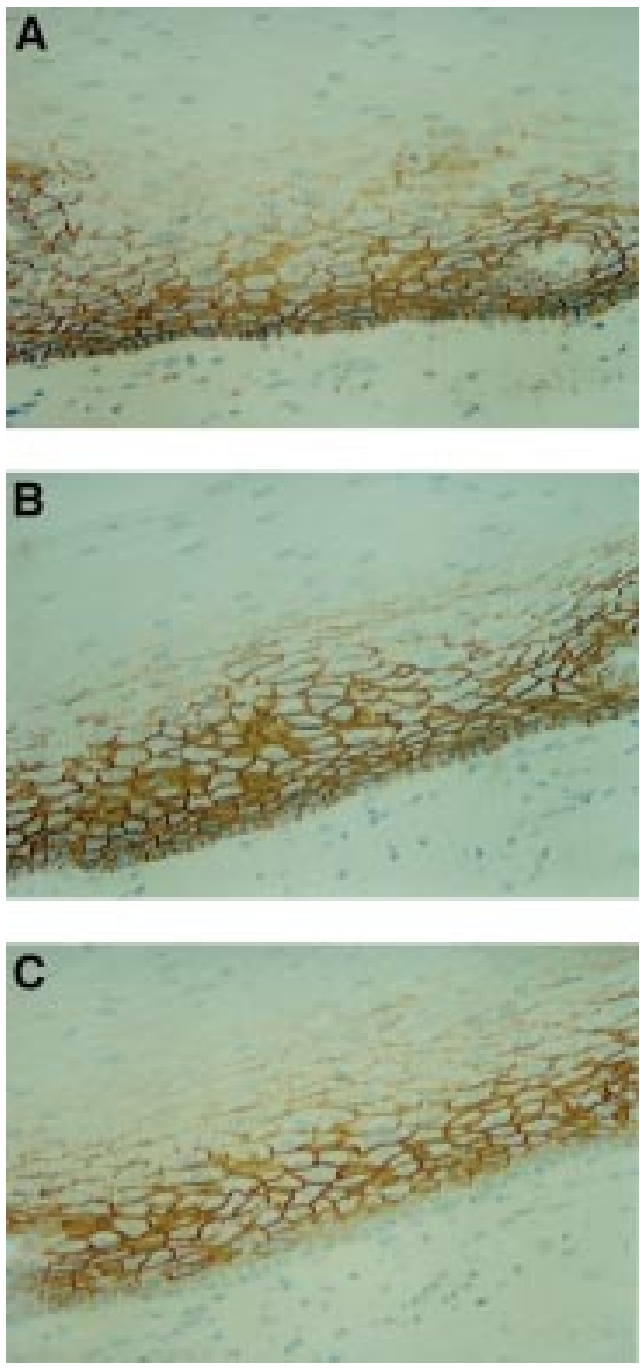

Figure 1 Immunohistochemistry of the adjacent normal squamous epithelium. (A) All normal squamous epithelium displayed uniform staining for total CD44 in the prickle cell and basal cell layers. $v 6(B)$ and $v 2(C)$ were also observed in the prickle cell and the basal cell layers. Normal mucosa adjacent to the cancer tissue provided positive internal controls for reliably assessing the presence or absence of CD44 immunoreactivity in the squamous cell carcinomas. Original magnification $\times 200$.

\section{Materials and methods}

PATIENTS AND TISSUE SPECIMENS

Samples of formalin fixed paraffin wax embedded tumour tissue were examined from 233 oesophageal squamous cell carcinomas curatively removed without additional treatment between 1987 and 1996 at the National Cancer Center Hospital, Tokyo. All patients underwent cervical, mediastinal, and abdominal lymphadenectomy (three field dissection) ${ }^{23}$ for oesophagectomy, and were classified according to the tumour, node, metastases (TNM) classification ${ }^{24}$ based on the pathological reports. The pathological stage (pTNM) of each patient was defined at the time of operation. The patients included 57 of pTNM stage I, 108 of stage II, and 68 of stage III. The 211 men and 22 women had a median age of 61 (range 40-86) years. The median follow up period was 33.7 (range 2-117) months.
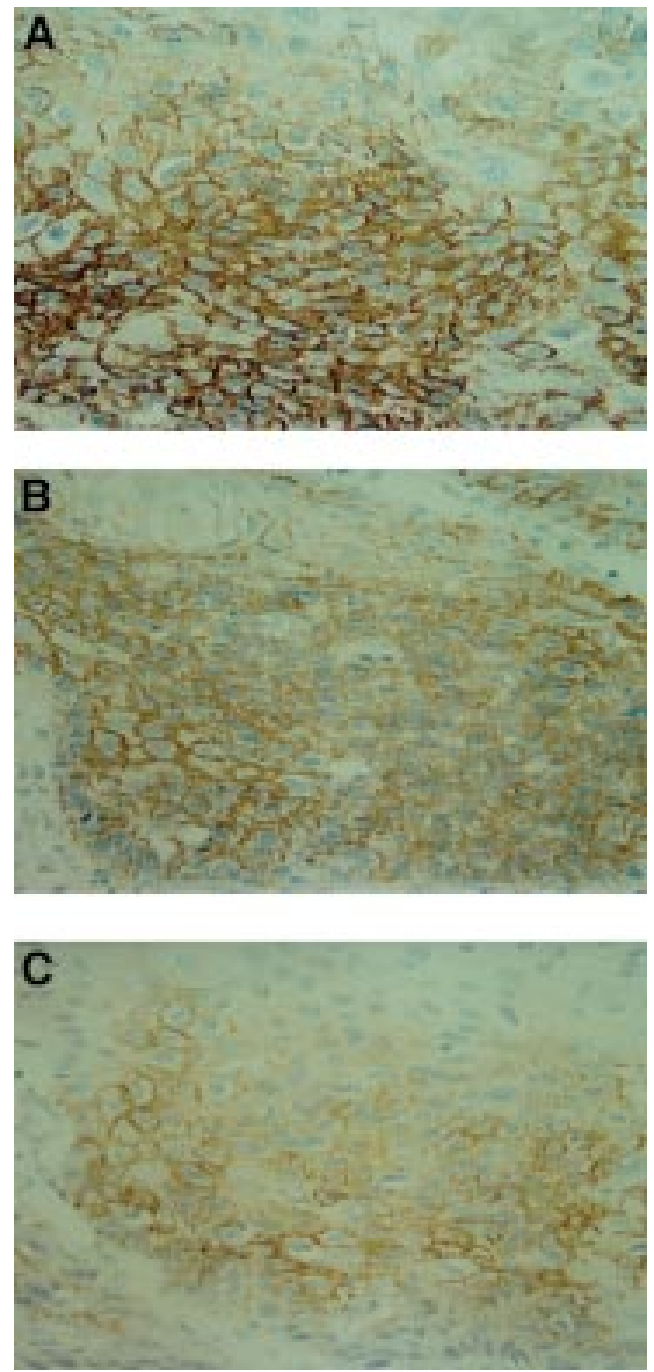

Figure 2 Immunohistochemistry of squamous cell carcinoma. Total CD44 (A) and v6 (B) were observed in 99\% (231 of 233) and 97\% (226 of 233) of the cancer specimens respectively. In contrast, the expression of v2 (C) in the cancer tissue was only 30\% (70 of 233). The intensity of v2 staining was generally weaker than that of total CD44 and v6 staining. Original magnification $\times 400$.

ANTIBODIES AND IMMUNOHISTOCHEMICAL ANALYSIS

Sections $(3 \mu \mathrm{m})$ were cut on silane coated glass slides (Muto Pure Chemicals, Tokyo, Japan), deparaffinised in xylene, dehydrated through a graded series of ethanols, and washed in running water. They were then treated with Serotec target unmasking fluid (STUF; Serotec, Oxford, UK) for 10 minutes in a $600 \mathrm{~W}$ microwave oven and left to cool for 15 minutes. They were rinsed in two changes of deionised water and phosphate buffered saline, and preincubated with $20 \%$ normal rabbit serum in Tris buffered saline at $37^{\circ} \mathrm{C}$ for 60 minutes. After being washed with Tris buffered saline, separate sections were incubated with the primary monoclonal antibodies 2C5 (3.3 $\mu \mathrm{g} / \mathrm{ml} ; \mathrm{R} \& \mathrm{D}$ Systems, Abingdon, Oxon, UK), 2F10 (10 $\mu \mathrm{g} / \mathrm{ml}, \mathrm{R} \& \mathrm{D}$ Systems), or $\mathrm{M} 23.6 .1^{20}(20 \mu \mathrm{g} / \mathrm{ml})$, which recognise epitopes of the CD44s, v6, and v2 portions respectively, in $1 \%$ normal rabbit serum diluted with Tris buffered saline at $4^{\circ} \mathrm{C}$ 
Table 1 Correlation between CD44v2 status and clinicopathological indexes

\begin{tabular}{|c|c|c|c|c|}
\hline & \multirow[b]{2}{*}{$n$} & \multicolumn{3}{|c|}{ CD44v2 expression } \\
\hline & & Positive & Negative & $p$ Value \\
\hline \multicolumn{5}{|l|}{ Sex } \\
\hline $\mathrm{M}$ & 211 & $64(30)$ & $147(70)$ & \multirow[t]{2}{*}{$0.96^{\mathrm{a}}$} \\
\hline $\mathrm{F}$ & 22 & $6(25)$ & $16(75)$ & \\
\hline Median age (years) & 61 & 61 & 61 & $0.71^{\mathrm{b}}$ \\
\hline \multicolumn{5}{|l|}{ Histological grading } \\
\hline Well, moderate & 191 & $61(32)$ & $130(68)$ & \multirow[t]{2}{*}{$0.19^{\mathrm{a}}$} \\
\hline Poor & 42 & $9(21)$ & $33(79)$ & \\
\hline \multicolumn{5}{|l|}{ Vessel invasion } \\
\hline$(-)$ & 164 & $53(32)$ & $111(68)$ & \multirow[t]{2}{*}{$0.88^{\mathrm{a}}$} \\
\hline$(+)$ & 69 & $17(25)$ & $52(75)$ & \\
\hline \multicolumn{5}{|l|}{ pT classification } \\
\hline $\mathrm{T} 1$ & 99 & $27(27)$ & $72(73)$ & \multirow[t]{3}{*}{$0.46^{\mathrm{a}}$} \\
\hline $\mathrm{T} 2$ & 61 & $17(28)$ & $44(72)$ & \\
\hline T3 & 73 & $26(37)$ & $47(63)$ & \\
\hline \multicolumn{5}{|l|}{ pN classification } \\
\hline No & 105 & $29(28)$ & $76(72)$ & \multirow[t]{2}{*}{$0.48^{\mathrm{a}}$} \\
\hline N1 & 128 & $41(32)$ & $87(68)$ & \\
\hline \multicolumn{5}{|l|}{ pTNM stage } \\
\hline I & 57 & $16(28)$ & $41(72)$ & \multirow{3}{*}{$0.15^{\mathrm{a}}$} \\
\hline II & 108 & $27(25)$ & $81(75)$ & \\
\hline III & 68 & $27(39)$ & $41(61)$ & \\
\hline \multicolumn{5}{|l|}{ Prognosis } \\
\hline Alive & 135 & $51(38)$ & $84(62)$ & \multirow[t]{2}{*}{$0.004^{\mathrm{a}}$} \\
\hline Dead & 98 & $19(19)$ & $79(81)$ & \\
\hline Median follow up periods (months) & 33.7 & 34.0 & 34.9 & $0.93^{\mathrm{b}}$ \\
\hline
\end{tabular}

Values in parentheses are percentages.

a Calculated by Fisher's test.

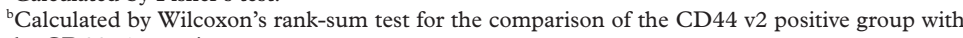
the CD44 v2 negative group.

overnight in a wet box and then at $37^{\circ} \mathrm{C}$ for 10 minutes the next morning. After the primary antibody treatment, endogenous peroxidase activity was blocked with $0.3 \% \quad \mathrm{H}_{2} \mathrm{O}_{2}$ in methanol at $4^{\circ} \mathrm{C}$ for 10 minutes, and the sections were incubated with a $1: 400$ dilution of biotinylated anti-mouse IgG (Dako, Santa Barbara, California, USA). This was followed by incubation with horseradish peroxidase conjugated avidin-biotin complex (ABComplex; Dako) at room temperature for 60 minutes. Immunostaining was visualised with 3,3'-diaminobenzidine (Sigma, St Louis, Missouri, USA) for 20 minutes, and the reaction was stopped by washing with water. Finally, the sections were counterstained with Mayer's haematoxylin. Between all antibody incubations, they were washed three times for five minutes with Tris buffered saline on a shaking platform. Negative controls included sections treated with $1 \%$ normal rabbit serum alone in place of the primary antibody.

The status of CD44s and CD44v was assessed in a coded manner without knowledge of the clinical and pathological features of the case or the clinical outcome. Levels of CD44 protein expression were graded into four subgroups: (a) no detectable expression; (b) faint expression detected in $<5 \%$ of tumour cells; (c) strong heterogeneous or localised

Table 2 Multivariate analysis with Cox's proportional hazards model for CD44v2 status and clinicopathological indexes in all patients studied

\begin{tabular}{llll}
\hline & $H R$ & $95 \%$ CI & p Value \\
\hline CD44 v2 status (negative $v$ positive) & 1.946 & 1.165 to 3.250 & 0.011 \\
pN (positive $v$ negative) & 2.226 & 1.355 to 3.659 & $<0.001$ \\
pT $(1 v 2$ and 3) & 2.003 & 1.240 to 3.236 & $<0.005$ \\
Age $(\leqslant 61 v>61)$ & 1.441 & 0.578 to 3.589 & 0.433 \\
Sex (male $v$ female) & 1.668 & 0.765 to 3.638 & 0.198 \\
Histology (poor $v$ well and moderate) & 1.248 & 0.774 to 2.013 & 0.363 \\
Vessel invasion (positive $v$ negative) & 1.227 & 0.684 to 2.202 & 0.492
\end{tabular}

HR, hazard ratio. expression in $>5 \%$ of tumour cells, indicating immunopositive subpopulation; (d) strong homogeneous expression in $>50 \%$ of tumour cells. For practical and statistical purposes, subgroups $(a)$ and $(b)$ were combined into the group designated "negative", and subgroups $(c)$ and $(d)$ were combined into the group designated "positive".

\section{STATISTICAL ANALYSIS}

Staining results were compared in all cases with clinicopathological features, including sex, age, pTNM classification, histological grading, vessel invasion, and survival. Each lesion was graded histologically according to the World Health Organisation classification. ${ }^{25}$ The correlation between CD44 peptide expression and clinicopathological indices was analysed by Fisher's exact test. We defined overall survival as survival from the date of surgery until 26 December 1996 . The primary determinant in this study was survival time, as measured from the date of surgery until the time of the last follow up visit or death. Survival curves for 233 patients were constructed according to the Kaplan-Meier method, ${ }^{26}$ and the differences between the curves were calculated. The $p$ value was calculated by the log rank analysis. The prognostic significance of individual parameters in multiparametric analyses was determined by using Cox's proportional-hazards model. ${ }^{27}$ A p value of less than 0.05 was considered significant.

\section{Results}

IMMUNOHISTOCHEMICAL STAINING

All 233 oesophageal squamous cell carcinomas were stained with three monoclonal antibodies directed against the total CD44 (CD44v as well as CD44s), v6 (exon 11), and v2 (exon 7). Moreover, normal oesophageal mucosa adjacent to cancer tissue was examined for reactivity with the same antibodies. All normal squamous epithelium displayed uniform staining for total CD44, v6, and v2 in the prickle cell and basal cell layers (fig $1 \mathrm{~A}-\mathrm{C})$. Normal mucosa adjacent to the cancer tissue provided positive internal controls for reliably assessing the presence or absence of CD44 immunoreactivity in the squamous cell carcinomas. Total CD 44 and v6 were observed in $99 \%$ (231 of 233) and 97\% (226 of 233) of the cancer specimens respectively (fig $2 \mathrm{~A}, \mathrm{~B}$ ). The high immunoreactivity to total CD44 and v6 was not only shown in normal squamous epithelium but also in cancer tissue. In contrast, the expression of $\mathrm{v} 2$ in the cancer tissue was only $30 \%$ (70 of 233). The intensity of v2 staining was generally weaker than that of total CD44 and v6 staining. Reactivity with all the CD44 specific antibodies was localised mainly on the tumour cell membranes, and some cells showed positive staining in the cytoplasm of the cells, especially in the case of CD44 v2. Table 1 summarises the v2 staining status of the 233 patients. 
Table 3 Multivariate analysis with Cox's proportional hazards model for CD44v2 status and clinicopathological indexes in patients without lymph node metastasis

\begin{tabular}{llll}
\hline & $H R$ & $95 \%$ CI & p Value \\
\hline CD44 v2 status (negative $v$ positive) & 4.729 & 1.108 to 20.183 & 0.036 \\
pT $(1$ 2 and 3) & 1.487 & 0.609 to 3.630 & 0.758 \\
Histology (poor $v$ well and moderate) & 1.105 & 0.356 to 3.430 & 0.863 \\
Vessel invasion (positive $v$ negative) & 0.973 & 0.385 to 2.455 & 0.954 \\
\hline
\end{tabular}

CORRELATION BETWEEN THE EXPRESSION OF V2 AND CLINICOPATHOLOGICAL INDICES

Table 1 gives the relevant clinical characteristics of the 233 patients whose tumours were analysed immunohistochemically. Neither sex nor age correlated with positivity for $\mathrm{v} 2$ ( $\mathrm{p}=$ 0.96 and 0.71 respectively). Also, there was no significant correlation between the expression of v2 and other clinicopathological indices such as histological grading, vessel invasion, pT classification, $\mathrm{pN}$ classification, or pTNM stage. The median follow up period was 34.0 months for patients with v2 positive tumours and 34.9 months for those with v2 negative tumours $(\mathrm{p}=0.93)$.

EXPRESSION OF V2 AND PROGNOSIS

The relation between the expression of $\mathrm{v} 2$ and survival rate was examined in 233 curatively resected oesophageal squamous cell carcinomas during a follow up period of 2-117 months (median 33.7 months). Figure 3 shows survival curves of patients, stratified according to v2 status. Patients whose tumours were v 2 positive had a significantly better prognosis than those whose tumours were v2 negative $(\mathrm{p}=0.031)$. The five year survival rate for patients with $\mathrm{v} 2$ positive tumours was $72.4 \%$, whereas the rate was $43.8 \%$ for patients with v2 negative tumours. At the conclusion of the study, 51 of 70 patients with v2 positive tumours (73\%) were alive, compared with 84 of 163 patients $(52 \%)$ with v2 negative tumours ( $p=0.004)$. In multivariate analysis with Cox's proportional hazards model, v2 status was an independent prognostic factor (relative risk of death associated with v2 negativity, $2.0 ; \mathrm{p}=$ 0.008) in all patients studied. However, the hazard ratio was lower than that of p T classification (relative risk of death associated with

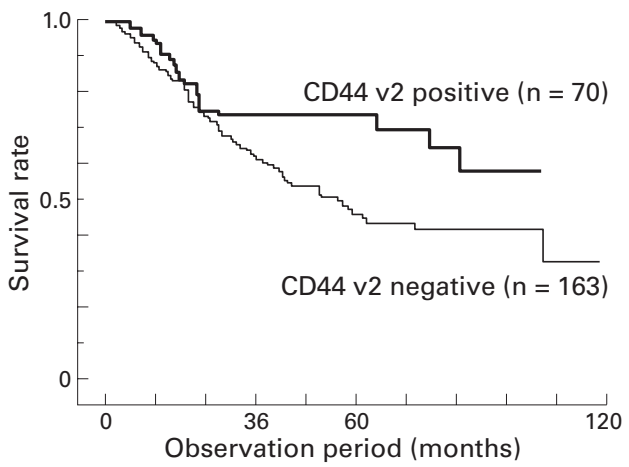

Figure 3 Survival curves of all patients studied according to v2 status. Patients whose tumours were v2 positive had a significantly better prognosis than those whose tumours were $v 2$ negative (log rank test; $p=0.031$ ). The five year survival rate for patients with $v 2$ positive tumours (median follow up period 34.0 months) was $72.4 \%$, whereas it was $43.8 \%$ for patients with $v 2$ negative tumours (median follow up period 34.9 months).

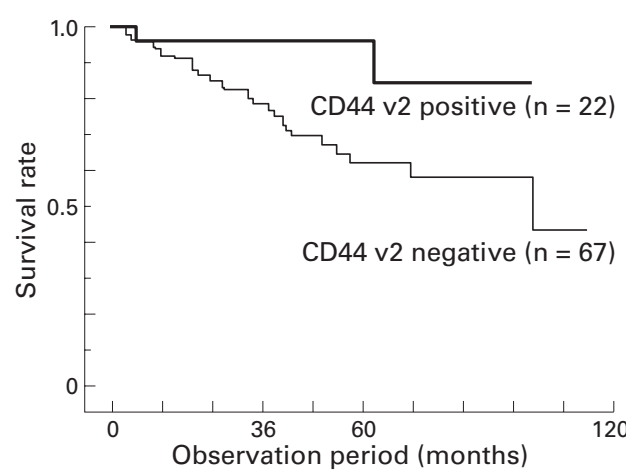

Figure 4 Survival curves of patients without lymph node metastasis according to 22 status. Patients whose tumours were $v 2$ positive had a significantly better prognosis than those whose tumors were $v 2$ negative (log rank test; $p=$ $0.022)$. The five year survival rate for patients with $v 2$ positive tumours (median follow up period 45.9 months) was $83.8 \%$, whereas it was $65.1 \%$ for patients with $v 2$ negative tumours (median follow up period 43.0 months).

pT2 and 3, 2.3; p = 0.003) or $\mathrm{pN}$ classification (relative risk of death associated with $\mathrm{pN} 1,2.6$; $\mathrm{p}<0.001)$. Other indices such as age, sex, histological grading and vessel invasion were not significant independent indicators of prognosis.

Figure 4 shows survival curves for patients without lymph node metastasis according to v2 status. As in the data from node negative patients studied, patients whose tumours were v2 positive had a significantly better prognosis than those whose tumours were $\mathrm{v} 2$ negative ( $\mathrm{p}$ $=0.022)$. The five year survival rate for patients with $\mathrm{v} 2$ positive tumours (median follow up period 45.9 months) was $83.8 \%$, whereas the rate was $65.1 \%$ for patients with v2 negative tumours (median follow up period 43.0 months). In contrast with the data from all patients including node positivity and negativity, the multivariate analysis with Cox's proportional hazards model showed that only v2 status was an independent prognostic factor (relative risk of death associated with v2 negativity, $4.7 ; \mathrm{p}=0.037$ ) in the patients without lymph node metastasis. However, pT classification, histological grading, and vessel invasion were not significant prognostic indicators. Tables 2 and 3 give the maximum likelihood estimated from the multivariate analysis.

\section{Discussion}

Recent clinicopathological studies have disclosed that the expression of individual variant exons is associated with poorer prognosis in several adenocarcinomas-for example, that of exon 14 (v9) is increased in gastric adenocarcinoma, ${ }^{28}$ that of exon 11 (v6) in the colon, breast, ${ }^{29} 30$ and pancreas, ${ }^{22} 3132$ and that of exon 7 (v2) in the pancreas ${ }^{21}$ and breast. $^{22}$ On the other hand, several reports have provided data indicating that CD44 expression is associated with longer survival in neuroblastoma ${ }^{33}$ and that decreased expression of v6 correlates with decreased survival in laryngeal squamous carcinoma ${ }^{34}$ and tumours of squamocellular origin. ${ }^{35}$ It is difficult to interpret this large body of 
information and to compare the various studies. However, we can infer from many reports published previously that the promoter and splice control is strictly regulated in normal cells in an organ specific manner, whereas the strict promoter and splice control is lost or changed in many human cancers. To date, there have been no reports on correlations between clinicopathological indices and CD 44v in oesophageal cancer. On the basis of this background, we studied the correlation between the expression of CD44v, especially $\mathrm{v} 2$, and survival of patients with squamous cell carcinoma.

In our study, all normal squamous epithelium displayed uniform staining of total CD44, v6, and v2 in the prickle cell and the basal cell layers. This result was consistent with other data for tumours of squamocellular origin. ${ }^{33-35}$ Whereas total CD44 and v6 were observed in $99 \%$ and $97 \%$ of the cancer specimens respectively, the expression of $\mathrm{v} 2$ in the cancer tissue was only $30 \%$. Furthermore, the intensity of v2 immunoreactivity was generally weaker than that of total CD44 and v6. Also, no significant association was found between the expression of $\mathrm{v} 2$ and clinicopathological indices of patients, including all stages. Concerning prognosis, patients with v2 positive tumours had a longer survival time than those with v2 negative tumours $(p=0.031)$. This result suggested that downregulation of $\mathrm{v} 2$ was associated with a poor prognosis in patients with oesophageal squamous cell carcinoma. In multivariate results of all patients studied, whereas v2 status was an independent prognostic factor (relative risk of death associated with v2 negativity, 2.0; $\mathrm{p}=0.008$ ), the hazard ratio was lower than that of $\mathrm{pT}$ classification or $\mathrm{pN}$ classification. As expected, the $\mathrm{pN}$ classification was an important prognostic factor ${ }^{36-38}$ (relative risk of death associated with $\mathrm{pN} 1$, $2.6 ; \mathrm{p}<0.001)$.

We then evaluated the association between v2 status and prognosis in the patients without lymph node metastasis, which was the most useful prognostic factor. The result showed not only that $\mathrm{v} 2$ positivity gave a significantly better prognosis $(p=0.022)$ but also that $v 2$ negativity was predictive of mortality (relative risk of death associated with v2 negativity, 4.7; $p=0.037)$ in the group of node negative patients. Other indices such as pT classification, pTNM stage, histological grading, and vessel invasion were not significant prognostic indicators.

To predict future development of metastasis is of great importance, as patients in such categories may benefit from adjuvant chemotherapy and/or radiotherapy. However, there has been no clear prognostic marker for patients without lymph node metastasis. Our findings here therefore seem to be quite important, because v2 status may assist in determining whether adjuvant therapy should be given to patients with oesophageal squamous cell carcinoma at an early stage.
We thank Miss S Nakadaira and Miss K Kobayashi for technical assistance and Miss H Orita for preparing the manuscript. This work was supported in part by the Grants-in-Aid for Cancer Research from the Ministry of Health and Welfare, Japan, the Japanese Foundation for Multidisciplinary Treatment of Cancer, and the Research Fund for Digestive Molecular Biology.

1 Haynes BF, Telen MJ, Hale LP, et al. CD44: a molecule nvolved in leukocyte adherence and T-cell activation. Immunol Today 1989;10:423-8.

2 Haynes BF, Liao HX, Patton KL. The transmembrane hyaluronate receptor (CD44): multiple forms. Cancer Cells 1991;3:347-50.

3 Stamenkovic I, Amiot M, Pesando JM, et al. Lymphocyte molecule implicated in lymph node homing is a member of the cartilage link protein family. Cell 1989;56:1057-62.

4 Goldstein LA, Zhou DFH, Picker LJ, et al. A human lymphocyte homing receptor, the Hermes antigen, is related to cartilage proteoglycan core and link proteins. Cell 1989;56:1063-72.

5 Jalkanen S, Bargatze RF, del los Toyos J, et al. Lymphocyte recognition of high endothelium: antibodies to distinctly epitopes of an 85-95 kD glycoprotein antigen differentially inhibit lymphocyte binding to lymph node, mucosal or inhibit lymphocyte binding to lymph node, muco

6 Aruffo A, Stamenkovic I, Melnick M, et al. CD44 is the Aruffo A, Stamenkovic I, Melnick M, et al. CD44 is the
principle cell surface receptor for hyaluronate. Cell 990;61:1303-13.

7 Arch R, Wirth K, Hofmann M, et al. Participation in normal immune responses of a metastasis-inducing splice variant of CD44. Science 1992;257:682-5.

8 Gunthert U, Hofmann M, Rudy W, et al. A new variant of glycoprotein CD44 confers metastatic potential to rat carcinoma cells. Cell 1991;65:13-24.

9 Matsumura Y, Hanbury D, Smith L, et al. Non-invasive detection of malignancy by identification of unusual CD44 gene activity in exfoliated cancer cells. BMF 1994;308:61924.

10 Matsumura Y, Sugiyama M, Matsumura S, et al. Unusual retention of introns in CD44 gene transcripts in bladder cancer provides new diagnostic and clinical oncological cancer provides new diagnostic and clini
opportunities. F Pathol 1995;177:11-20.

11 Screaton GR, Bell MV, Jackson DG, et al. Genomic structure of DNA encoding the lymphocyte homing recepstructure of DNA encoding the lymphocyte homing receptor CD44 reveals at least 12 alternatively
Proc Natl Acad Sci USA 1992;89:12160-4.

12 Tolg C, Hofmann M, Herrlich P, et al. Splicing choice from ten variant exons establishes CD44 variability. Nucleic Acids Res 1993;21:1225-9.

13 MacKay CR, Terpe HJ, Stauder R, et al. Expression and modulation of CD 44 variant isoforms in human. $\mathcal{F ~ C e l l ~ B i o l ~}$ 1994;124:71-82.

14 Yamao T, Matsumura Y, Shimada Y, et al. Abnormal expression of CD44 variants in the exfoliated cells in the feces of patients with colorectal cancer. Gastroenterology 1998;114:1196-205.

15 Hoffmann M, Rudy W, Zoller M, et al. CD44 splice variants confer metastatic behavior in rats: homologous sequences are expressed in human tumor cell lines. Cancer Res 1991; 51:5292-7.

16 Matsumura Y, Tarin D. Significance of CD44 gene products Matsumura Y, Tarin D. Significance of CD44 gene products
for cancer diagnosis and disease evalution. Lancet 1992; 340:1053-8.

17 Tanabe K, Elis LM, Saya H. Expression of CD44R1 adhesion molecule in colon carcinomas and metastasis. Lancet 1993;341:725-6.

18 Heider KH, Hoffmann M, Horst E, et al. A human homologue of the rat metastasis associated variant of CD44 is expressed in colorectal carcinomsa and adenomatous polyps. F Cell Biol 1993;120:227-33.

9 Heider KH, Dammrich J, Skroch-Angel P, et al. Differential expression of CD44 splice variants in intestinal- and diffuse-type human gastric carcinomas and normal gastric mucosa. Cancer Res 1994;53:4197-203.

20 Borgya A, Woodman A, Sugiyama M, et al. Isolation and characterization of antibodies which specifically recognize the peptide encoded by exon 7 (v2) of the human CD44 gene. F Clin Pathol Mol Pathol 1995; 48:M241-50.

21 Tokue Y, Matsumura Y, Katsumata N, et al. CD44 variant isoform expression and breast cancer prognosis. fpn $\mathcal{f}$ Cancer Res 1998;89:283-90.

22 Gotoda T, Matsumura Y, Kondo H, et al. Expression of Gotoda $\mathrm{T}$, Matsumura $\mathrm{Y}$, Kondo $\mathrm{H}$, et al. Expression of CD44 variants and its association with survival in
atic cancer. Fpn f Cancer Res 1998;89:1033-40.

23 Isono K, Sato H, Nakayama K. Results of nationwide study on the three-field lymph-node dissection of esophageal cancer. Oncology 1991;48:411-20.

24 Hermanek P, Sobin LH. TNM classification of malignant tumors, 4th ed. Berlin: Springer, 1987

25 Watanabe H, Jass JR, Sobin LH. Histological typing of oesophageal and gastric tumours, 2nd ed. Berlin: Springer, 1990.

26 Kaplan EL, Meier P. Nonparametric estimation from incomplete observations. Fournal of the American Statistics Association 1958;53:457-81.

27 Cox DR. Regression models and life tables. Fournal of the Royal Statistical Society 1972;24:187-20.

28 Mayer B, Jauch KW, Gunthert U, et al. De-novo expression Mayer B, Jauch KW, Gunthert U, et al. De-novo expression
of CD-44 and survival in gastric cancer. Lancet 1993;342: of CD-44.

29 Mulder JW, Kruyt PM, Sewnath M, et al. Colorectal cancer prognosis and expression of exon-v6-containing CD44 proteins. Lancet 1994;344:1470-2. 
30 Kaufmann M, Heider KH, Sinn HP, et al. CD44 variant exon epitopes in primary breast cancer and length of exon epitopes in primary breast
survival. Lancet 1995;345:615-19.

31 Rall CJN, Rustgi AK. CD44 isoform expression in primary and metastatic pancreatic adenocarcinoma. Cancer Res 1995;55:1931-5.

32 Castella EM, Ariza A, Ojanguren I, et al. Differential expression of CD44v6 in adenocarcinoma of the pancreas: an immunohistochemical study. Virchows Arch 1996;429: $191-5$

33 Favrot MC, Combaret V, Lasset C. CD44: a new prognostic marker for neuroblastoma. N Engl F Med 1993;329:1965.

34 Spafford MF, Koeppe I, Pan Z, et al. Correlation of tumor markers p53, bcl-2, CD $34, \mathrm{CD} 44 \mathrm{H}, \mathrm{CD} 44 \mathrm{v} 6$, and $\mathrm{Ki}-67$ with survival and metastasis in laryngeal squamous cell. Arch Otolaryngol Head Neck Surg 1996;122:627-32.
35 Salmi M, Gron-Virta K, Sointu P, et al. Regulated expression of exon v6 containing isoforms of CD44 in man: downregulation during makignant transformation of tumors of squamocellular origin. $\mathcal{F}$ Cell Biol 1993;122:43142.

36 Kato $\mathrm{H}$, Tachimori $\mathrm{Y}$, Watanabe $\mathrm{H}$, et al. Evaluation of the new (1987) TNM classification for thoracic esophageal tumors. Int 7 Cancer 1993;53:220-3.

37 Ohbu M, Saegusa M, Kobayashi H, et al. Expression of $b c l-2$ protein in esophageal squamous cell carcinomas and its association with lymph node metastasis. Cancer 1997;79: $1287-93$.

38 Kawahara K, Maekawa T, Okabayashi K, et al. The number of lymph node metastases influences survival in esophageal cancer. F Surg Oncol 1998;67:160-3. 